\title{
Severe Isolated Thrombocytopenia Associated with the Use of Clopidogrel
}

\author{
Yingying Zhang1, Hongyan Zhou' ${ }^{2}$, Wei Han ${ }^{*}$ \\ ${ }^{1}$ Department of Cardiology, The First Affiliated Hospital of Harbin Medical University, Harbin, China \\ ${ }^{2}$ Fuwai Hospital, Beijing, China \\ Email: ${ }^{*}$ medhan@126.com
}

Received 14 January 2014; revised 13 February 2014; accepted 12 March 2014

Copyright (c) 2014 by authors and Scientific Research Publishing Inc.

This work is licensed under the Creative Commons Attribution International License (CC BY).

http://creativecommons.org/licenses/by/4.0/

c) (i) Open Access

\begin{abstract}
Clopidogrel is used as a frontline antiplatelet drug in patients with coronary artery disease, peripheral vascular disease and cerebrovascular disease. Though it is frequently reported that clopidogrel is associated with thrombotic thrombocytopenic purpura (TTP), severe isolated thrombocytopenia induced by this drug is rare. Here we report a case with isolated thrombocytopenia due to clopidogrel therapy. As this agent is widely used in clinical practice, the occurrence of this rare but dangerous adverse effect should be considered.
\end{abstract}

\section{Keywords}

\section{Clopidogrel, Antiplatelet, Thrombocytope}

\section{Introduction}

Thrombocytopenia followed by percutaneous coronary intervention (PCI) is related with the therapy of antithrombotic, mainly with the use of heparin, glycoprotein IIb/IIIa (GPIIb/IIIa) receptor inhibitors and ticlopidine. Clopidogrel, as ticlopidine, is a thienopyridine derivative, irreversibly inhibits platelet aggregation by selective binding of adenylate cyclase bound ADP receptors on the platelet surface, and it is considered safer than ticlopidine due to its decreased events of hematologic adverse effects. Although thrombotic thrombocytopenic purpura (TTP) is the most reported adverse effect of clopidogrel, isolated thrombocytopenia is another rare hematologic adverse effect [1]. However, the mechanism of isolated thrombocytopenia remains speculative. To the best of our knowledge, there are only four reports have involved this severe adverse effect induced by clopidogrel [2]-[5]. Here we report a case of a 73-year-old woman with unstable angina, treated with stent implantation, in whom acute stent thrombosis and twice thrombocytopenia were observed after PCI which is associated with clopidogrel-induced isolated thrombocytopenia.

${ }^{*}$ Corresponding author. 


\section{Case Presentation}

Our patient was a 73-year-old woman with a medical history of hypertension and hyperlipidemia, controlled with Benazepril Hydrochloride (10 mg/day, Novartis, Switzerland) and atorvastatin (20 mg/day, Pfizer, USA). At presentation complete blood count revealed WBC $7.23 \times 10^{9} / \mathrm{L}$ with normal WBC differential conuts, hemoglobin $142 \mathrm{~g} / \mathrm{L}$ and platelet $197.4 \times 10^{9} / \mathrm{L}$. She had normal values in blood clotting, liver and renal functions. The patient had been started a regimen with aspirin (Bayer, Germany), clopidogrel (Sanofi-Aventis, France), imdur (Astrazeneca, Sweden), atorvastatin (Pfizer, USA) and benazepril hydrochloride (Novartis, Switzerland) for 5 days until catheterization. On December 18th, 2012, coronary angiography showed an 80\% diffuse lesion in the mid-left anterior descending artery (LAD), a 99\% lesion in the mid-left circumflex artery, and a 99\% lesion in the mid-posterior right ventricular branches. Percutaneous coronary intenvention (PCI) was performed and one lepu stent $(3.5 \times 36 \mathrm{~mm})$, one firebird stent $(2.5 \times 18 \mathrm{~mm})$ and one firebird stent $(3.0 \times 33 \mathrm{~mm})$ were placed on to mid-left anterior descending artery, mid-left circumflex artery, and mid-posterior right ventricular branches respectively (Figure 1). There was also a 50\% lesion in the proximal left anterior descending artery without a stent The patient was treated with tirofiban $(10 \mu \mathrm{g} / \mathrm{kg}$ iv followed by $0.15 \mu \mathrm{g} / \mathrm{kg} / \mathrm{min}$ ivgtt, Grandpharma, China) and enoxaparin (0.4 ml Bid iH, Sanofi-Aventis, France) postoperatively.

The patient developed skin petechia around the site of puncture postoperatively, so we took a complete blood counts and the platelet count dropped to $36.2 \times 10^{9} / \mathrm{L}$. Pseudo thrombocytopenia was excluded. Meanwhile, as the patient had no history of heparin exposure in the last three months and administrated heparin with a brief period in this treatment, as well as serological testing for heparin induced thrombocytopenia was negative, heparin-induced thrombocytopenia (HIT) was also excluded. Nine hours after primary PCI, the patient complained of severe chest pain with diaphorisis and ECG showed characteristic signs of acute ST-elevation in leads I, aVL and V1 - V6. Coronary angiography of the left anterior descending artery showed a complete thrombotic occlu-

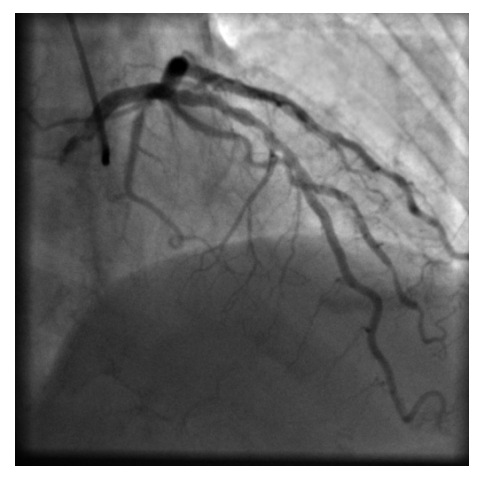

(a)

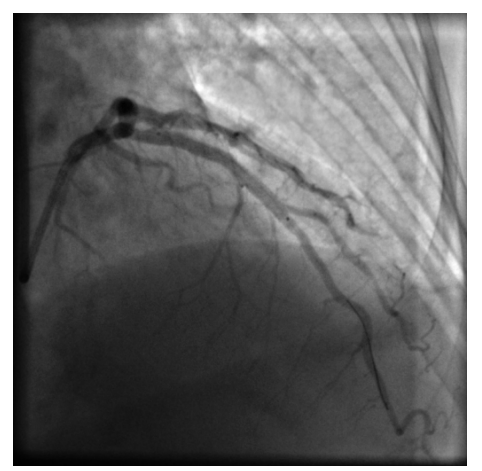

(d)

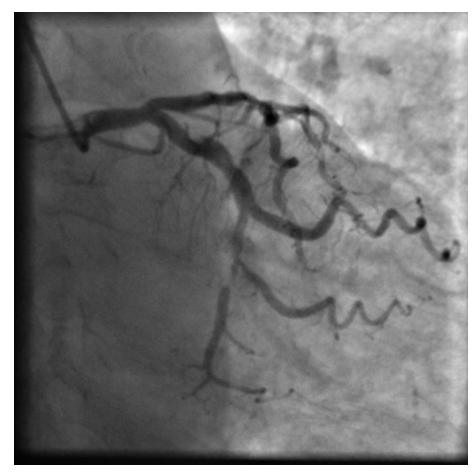

(b)

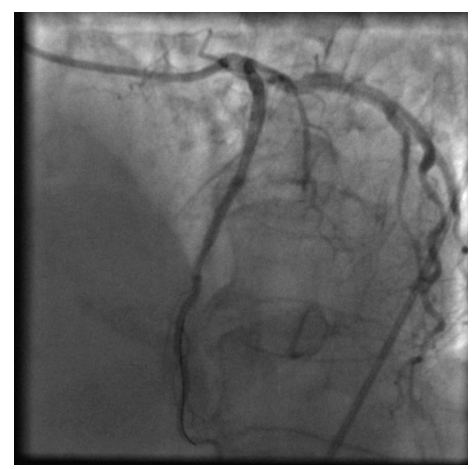

(e)

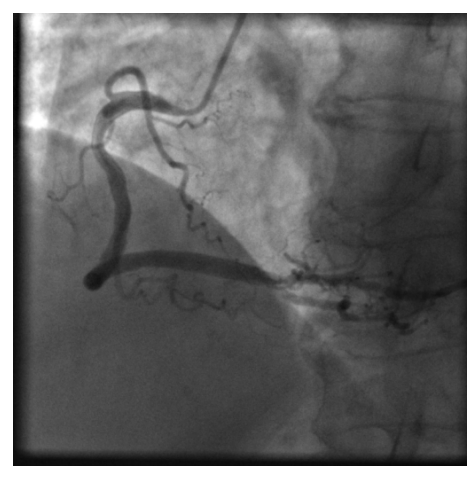

(c)

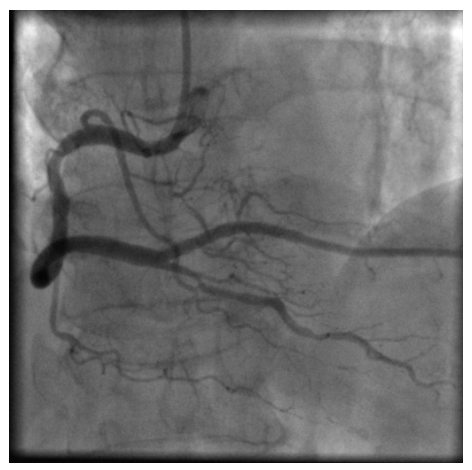

(f)

Figure 1. Coronary angiography showed an $80 \%$ diffuse lesion in the mid-left anterior descending artery (a), a $99 \%$ lesion in the mid-left circumflex artery (b), and a $99 \%$ lesion in the mid-posterior right ventricular branches (c). Percutaneous coronary intenvention (PCI) was performed and one lepu stent (3.5 × $36 \mathrm{~mm}$, (d)), one firebird stent $(2.5 \times 18 \mathrm{~mm}$, (e) $)$ and one firebird stent $(3.0 \times 33 \mathrm{~mm}$, (f)) were placed on to mid-left anterior descending artery, mid-left circumflex artery, and mid-posterior right ventricular branches respectively. 
sion with trombolysis in myocardial infarction (TIMI) 0 flow (Figure 2). The right coronary artery and the left circumflex artery appeared to be normal. The thrombus was aspirated and dissolved with urokinase 250 thousand unit within LAD. Another firebird stent $(3.0 \times 18 \mathrm{~mm})$ was placed in distal LAD resulting in a TIMI III flow. The chest pain was relieved and postprocedural electrocardiogram showed resolution of ST segment elevation. Thrombelastography (TEG) analysis showed MA was $84 \mathrm{~mm}$, R was $20.2 \mathrm{~min}$, so enoxaparin was stopped. With highly suspected that the severe thrombocytopenia is associated with tirofiban, tirofiban was also stopped. Aspirin and clopidogrel were continued after operation.

Fifteen hours after the second operation, the patient developed excruciating chest pain again. The ECG recorded significant ST-segment elevations over leads I, aVL and V1 to V6. The patient received fibrinolytic therapy with alteplase and her symptom obviously relieved and ST-segment elevation fall back more than $50 \% 2 \mathrm{~h}$ later. However, the patient developed massive hemorrhage of upper gastrointestinal tract, hematomas at the site of puncture and the platelet count decreased to $31 \times 10^{9} / \mathrm{L}$ (Figure 3), hemoglobin dropped to $119 \mathrm{~g} / \mathrm{L}$ and D-dimer increased to $7820 \mathrm{ng} / \mathrm{ml}$. Tirofiban-associated thrombocytopenia is also excluded. We confirmed that the symptom is induced by the use of clopidogrel, so clopidogrel is immediately discontinued, and replaced by cilostazol. Antiplatelet antibodies were negative, clopidogrel-induced immune thrombocytopenia can be ruled out. Blood bilirubin and coagulation function assays were normal and a bone marrow biopsy showed an actively proliferating marrow with increased megakaryocytes but no platelet-generated megakaryocytes. The patient's renal function is normal and in peripheral blood smear there is no found of red cell schistocytosis, TTP was excluded. The platelet count increased gradually and upto $156 \times 10^{9} / \mathrm{L}$ and D-dimer decreased to $5042 \mathrm{ng} / \mathrm{ml}$ (Figure 4) on the eleventh day. The diagnosis of clopidogrel-associated isolate thrombocytopenia is established.

The patient has normal platelet count and no bleeding and adverse coronary events happened in one month follow-up.

\section{Discussion}

There are several possible reasons for thrombocytopenia after PCI. In case of platelet decrease, firstly, we must exclude pseudothrombocytopenia. Our patient presented with skin petechia, this option is ruled out. Secondly, real thrombocytopenia can be divided into inherited and acquired, as our patient is a 73 years old woman with no medical history of thrombocytopenia, it can be suggested as an acquired form of thrombocytopenia [6]. Thirdly, acquired thrombocytopenia can be divided into immune and nonimmune mediated. Drugs are a common cause of acute immune-mediated thrombocytopenia, and most cases of drug-induced thrombocytopenia are caused by drug-dependent antibodies [7]. In the present case, HIT antibodies and antiplatelet antibodies were negative, so heparin-induced thrombocytopenia (HIT) and other drugs induced immune-mediated thrombocytopenia were excluded. Drugs can also cause nonimmune thrombocytopenia, inhibiting bone marrow thrombopoiesis. Our patient's platelet count continued rising after the discontinued of clopidogrel without any other therapies, and did not display chronically low, supporting the drug-induced thrombocytopenia.

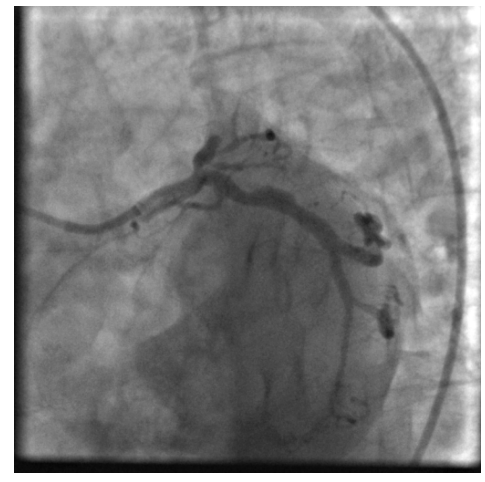

(a)

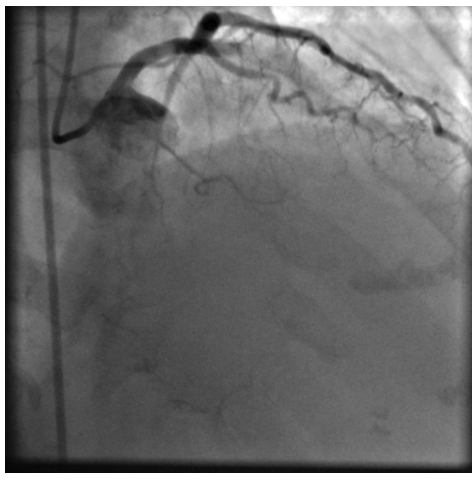

(b)

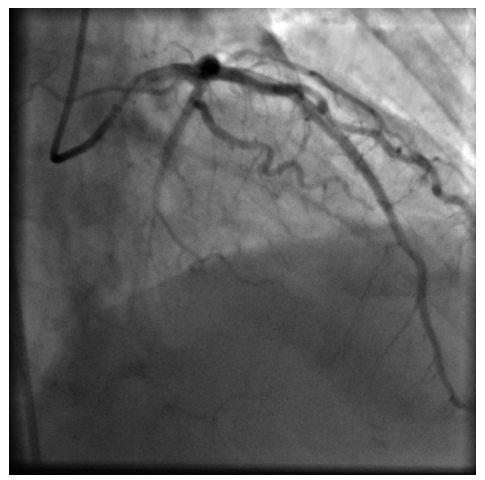

(c)

Figure 2. Coronary angiography of the left anterior descending artery showed a complete thrombotic occlusion with trombolysis in myocardial infarction (TIMI) 0 flow ((a), (b)). The thrombus was aspirated and dissolved with urokinase 250 thousand unit within LAD. Another firebird stent $(3.0 \times 18 \mathrm{~mm})$ was placed in distal LAD resulting in a TIMI III flow (c). 

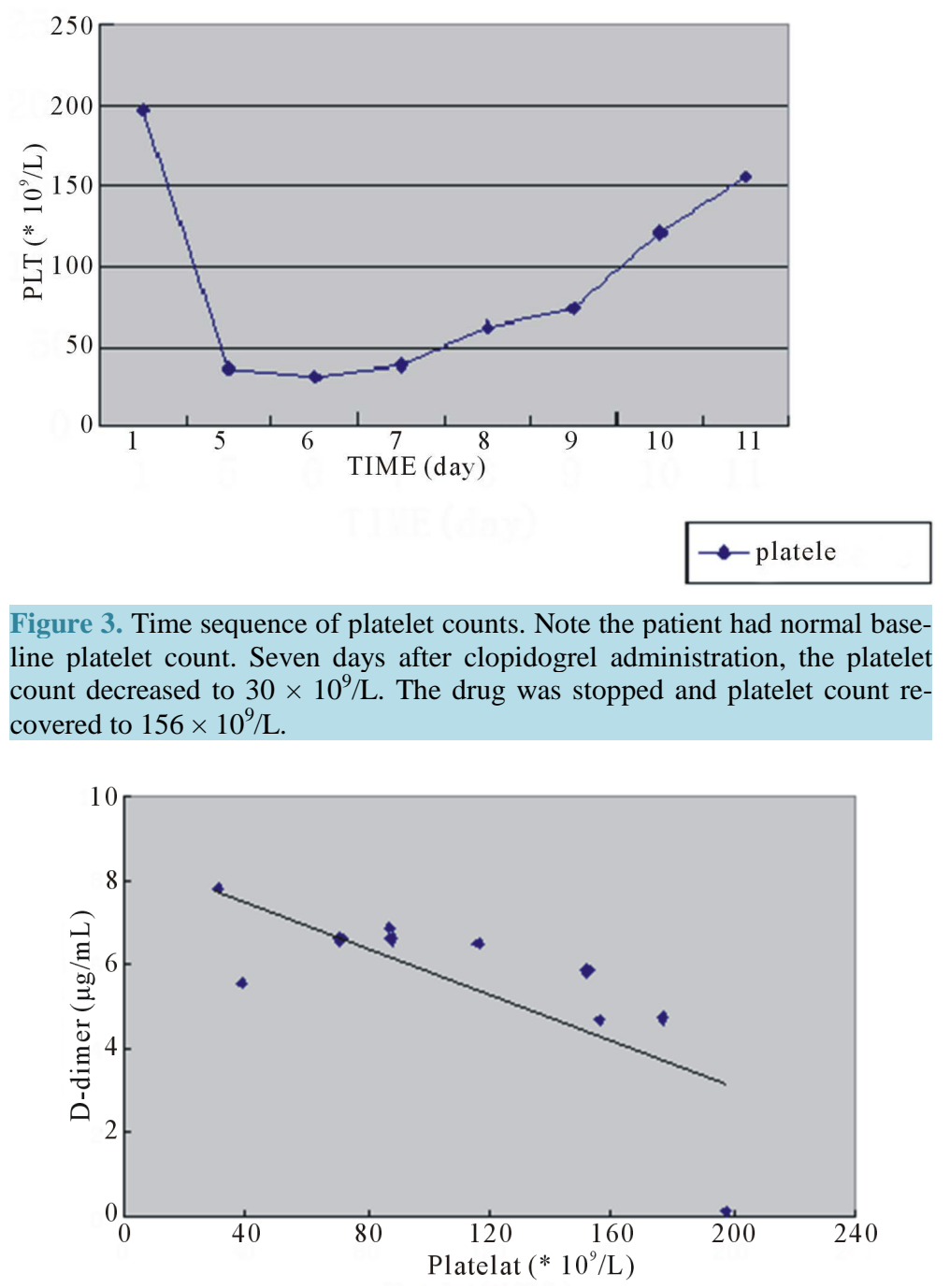

Figure 4. The inversed relationship between platelet account and Ddimer level.

TTP related to clopidegrel is the rare condition which comprises thrombocytopenia, microangiopathic hemolytic anemia, fever, neurological disorders and renal dysfunction. In this case, the anemia was not a microangiopathic hemolytic anemia, which was thought to be related to massive hemorrhage of upper gastrointestinal tract, and neither red cell schistocytes in peripheral blood smear nor abnormality in bone marrow were noted, suggesting not a microangiopathic hemolytic anemia. She had no renal abnormalities. More importantly, the platelet count recovered without plasma-exchange therapy meant that less probability the diagnosis was PPT. Thus, clopidogrel-associated isolate thrombocytopenia was diagnosed.

More importantly, there was an inversed relationship between platelet account and D-dimer level in the present case (Figure 4), suggesting thrombocytopenia associated with antiplatele drugs actually increased the risk for platelet activation and aggregation under some circumstance. Furthermore, TEG analysis showed R value was higher than normal and heparin was effective in the occurrence of stent thrombosis, documented an inadequate antiplatelet reaction and blood coagulation factors seems unlikely played a major role.

\section{Conclusions}

A combination antithrombotic and antiplatelet therapy with clopididogrel, aspisin, glycoprotein IIb/IIIa receptor inhibitors and low molecular weight heparin has been reported to be safe and effective in reducing major ad- 
verse cardiovascular events in patients undergoing PCI. Nevertheless, there is growing evidence that antithrombotic and antiplatelet drugs were related to thrombocytopenia. In this case, we see the severe isolated thrombocytopenia caused by clopidogrel, although, the exact mechanism of isolate thrombocytopenia remains unclear. When using these drugs, physicians should be aware that there are possibilities of drug-induced severe thrombocytopenia and platelet count should be monitored.

Large clinical studies revealed that thrombocytopenia associated with antiplatelet treatment not only increased risk of bleeding, but also thrombotic events [8]. For the first time, we demonstrated directly relationship between thrombocytopenia and thrombosis (indicated by D-dimer level). We are unaware of any prior report with similar observations. However, the underlying mechanisms need a further study.

\section{References}

[1] Balamuthusamy, S. and Arora, R. (2007) Hematologic Adverse Effects of Clopidogrel. American Journal of Therapeutics, 14, 106-112. http://dx.doi.org/10.1097/01.mjt.0000212708.81034.22

[2] Su, C.H., Tsai, C.F., Ueng, K.C., et al. (2004) Clopidogrel-Associated Severe Isolated Thrombocytopenia-A Case Report. Acta Cardiological Sinica, 20, 182-186.

[3] Elmi, F., Peacock, T. and Schiavone, J. (2000) Isolated Profound Thrombocytopenia Associated with Clopidogrel. Journal of Invasive Cardiology, 12, 532-535.

[4] Helft, G., Elalamy, I., Laudy, C., et al. (2003) Clopidogrel and Thrombopenia. A Case Report. Annales de Cardiologie et d'Angéiologie, 52, 191-193. http://dx.doi.org/10.1016/S0003-3928(02)00185-3

[5] da Silva Vedes, E.C., Marques, L.D.G. and Rico, M.C.T. (2011) Severe Isolated Thrombocytopenia after Clopidogrel and Pentoxifylline Therapy: A Case Report. Journal of Medical Case Reports, 5, 281. http://dx.doi.org/10.1186/1752-1947-5-281

[6] Drachman, J.G. (2004) Inherited Thrombocytopenia: When a Low Platelet Count Does Not Mean ITP. Blood, 103, 390-398. http://dx.doi.org/10.1182/blood-2003-05-1742

[7] George, J.N. and Aster, R.H. (2009) Drug-Induced Thrombocytopenia: Pathogenesis, Evaluation, and Management. Hematology American Society of Hematology Education Program, 2009, 153-158. http://dx.doi.org/10.1182/asheducation-2009.1.153

[8] Scirica, B.M., Cannon, C.P., Cooper, R., et al. (2006) Drug-Induced Thrombocytopenia and Thrombosis: Evidence from Patients Receiving an Oral Glycoprotein IIb/IIIa Inhibitor in the Orbofiban in Patients with Unstable Coronary Syndromes-(OPUS-TIMI 16) Trial. Journal of Thrombosis and Thrombolysis, 22, 95-102. 